\title{
Clinical Outcomes after Minimally Invasive Trans-Psoas Lateral Lumbar Interbody Fusion for the Treatment of Adult Degenerative Scoliosis: Four Years' Multicenter Study
}

\author{
Sheikh Asad ${ }^{1}$ Arjun Dubey ${ }^{1}$ Arvind Dubey ${ }^{1,2} \quad$ Chester Sutterlin ${ }^{1,2,3}$
}

\author{
${ }^{1}$ Department of Neurosurgery, Royal Hobart Hospital, Hobart, \\ Tasmania, Australia \\ ${ }^{2}$ Hobart Brain and Spine Centre, Hobart, Tasmania, Australia \\ ${ }^{3}$ Department of Neurosurgery, University of Florida, Gainesville, \\ Florida, United States
}

Indian J Neurosurg:2020;9:225-229

\begin{abstract}
Address for correspondence Sheikh Asad, MD, Department of Neurosurgery, Royal Hobart Hospital, Hobart, Tasmania, Australia (e-mail:dr.sheikhasad@gmail.com).
\end{abstract}

\begin{abstract}
The use of minimally invasive transpsoas lateral lumbar interbody fusion (LLIF) surgery for treatment of adult degenerative scoliosis is rapidly increasing in popularity. However, limited data is available regarding its use in adult degenerative lumbar scoliosis surgery. The objective of this study was to evaluate the clinical outcomes of adults with degenerative lumbar scoliosis who were treated with minimally invasive LLIF. Thirty-two consecutive patients with adult degenerative scoliosis treated by a single surgeon at two spine centers were followed up for an average of 13.2 months. Interbody fusion was completed using the minimally invasive LLIF technique with supplemental 360 degrees' posterior instrumentation. Oswestry disability index (ODI) scores were obtained preoperatively and at most recent follow-up. Complications were recorded. The study

Keywords

- lateral lumbar interbody fusion

- scoliosis

- trans-psoas group demonstrated improvement in clinical outcome scores. ODI scores improved from 36.8 to 23.4 ( $p<0.00001)$. A total of four complications $(12 \%)$ were recorded, and two patients $(6 \%)$ required additional surgery. Based on the significant improvement in validated clinical outcome scores, minimally invasive LLIF can be considered an effective procedure in the treatment of adult degenerative scoliosis.
\end{abstract}

\section{Introduction}

Lateral lumbar interbody fusion (LLIF) using a minimally invasive lateral retroperitoneal transpsoas approach was first reported by McAfee et al in 2006. ${ }^{1,2}$ Since then, it has been used for lumbar spine surgery in patients with degenerative spinal disorders, scoliosis, trauma, infections, and tumors.

Minimally invasive transpsoas lateral lumbar interbody fusion has several advantages such as ability to use wider foot print of interbody cage after maximal disc excision, indirect neural decompression, minimum blood loss, sparing of anterior or posterior longitudinal ligaments, shorter surgical time and hospital stay, cost effectiveness, and the effective correction of coronal balance. ${ }^{3-5}$

Prevalence of adult degenerative scoliosis cited in the literature ranges from 2.5 to $60 \%,{ }^{6}$ depending on severity. Patients classically present with back pain, sagittal imbalance, or radicular symptoms. Although conservative management is recommended as an initial treatment, outcomes are frequently unacceptable. ${ }^{6}$

When nonoperative treatment fails, adult degenerative scoliosis presents significant surgical challenges. Decompression may be the treatment of choice in mild
DOI https://doi.org/ 10.1055/s-0040-1710105 ISSN 2277-954X.

\footnotetext{
(C) 2020. Neurological Surgeons' Society of India.

This is an open access article published by Thieme under the terms of the Creative Commons Attribution-NonDerivative-NonCommercial-License, permitting copying and reproduction so long as the original work is given appropriate credit. Contents may not be used for commercial purposes, or adapted, remixed, transformed or built upon. (https://creativecommons.org/licenses/by-nc-nd/4.0/)

Thieme Medical and Scientific Publishers Pvt. Ltd., A-12, 2nd Floor, Sector 2, Noida-201301 UP, India
} 
deformity or minimal instability; typically, only used at one or two vertebral levels in patients with leg pain from stenosis and smaller curves (<30 degrees). However, decompression alone has been associated with a risk of iatrogenic instability and progression of deformity. For this reason, an instrumented arthrodesis such as LLIF with or without pedicle subtraction osteotomy (PSO) is often indicated., ${ }^{2,5}$

\section{Materials and Methods}

\section{Study Design}

This retrospective outcome analysis was conducted in patients who underwent LLIF for adult degenerative scoliosis and were treated by a single surgeon at two major academic institutions. During the study period, 32 consecutive patients underwent LLIF with supplemental posterior pedicle screw instrumentation and posterior lumbar interbody fusion (PLIF) when needed. The Oswestry disability index (ODI) is an index derived from the Oswestry low back pain questionnaire used by clinicians and researchers to quantify disability for low-back pain.

The self-completed questionnaire contains a set of questions with a range of score from 0 to 100 . Zero is equated with no disability and 100 is the maximum disability possible.

Validated clinical outcome scores were obtained preoperatively and at most recent follow-up for comparison purposes. Coronal balance angles were measured on preop and immediate postop X-ray ( - Figs. 1A, 2A). Further clinical follow-up was at 6,12 , and 24 months with flexion/extension X-rays. Dynamic lumbar spine X-rays were used in 30 out of 32 and CT scan in only 2 out of 32 patients to ascertain the degree of fusion. Complications were recorded.

\section{Subjects}

Thirty-two patients were followed up for an average of 13.2 months from 2012 to 2016 (-Table 1). Inclusion criteria required a diagnosis of symptomatic degenerative adult scoliosis that had failed at least a year of conservative treatment. Age range was 42 to 80 years. The study included 10 men and 22 women. Eleven patients were active smokers at the time of surgery. Average body mass index (BMI) was 29.5 (range 20-38.5).

\section{Surgical Technique}

Interbody fusion was completed using the LLIF technique (-Fig. 3). Laterally placed interbody spacers (Aleutian PEEK or Cascadia 3D printed titanium-K2M USA) were supplemented with i-Factor (Cerapedics USA) bone graft (-Fig. 1B). Lateral approaches were made from the left side which was often the convexity of the scoliotic curve. Posterior instrumentation involved placement of transpedicular screws and rods (-Figs.2B,4). A total of 71 levels from L1 to L5 (average of 2.2 levels per patient) were treated using LLIF. In addition to LLIF, traditional anterior interbody fusion (ALIF) or PLIF was used in some patients who required an L5-S1 fusion. Typically, all required procedures were performed during a single operative session. However, in patients requiring additional PLIF or ALIF, the posterior instrumentation portion of the case was performed 2 to 7 days after the LLIF portion.

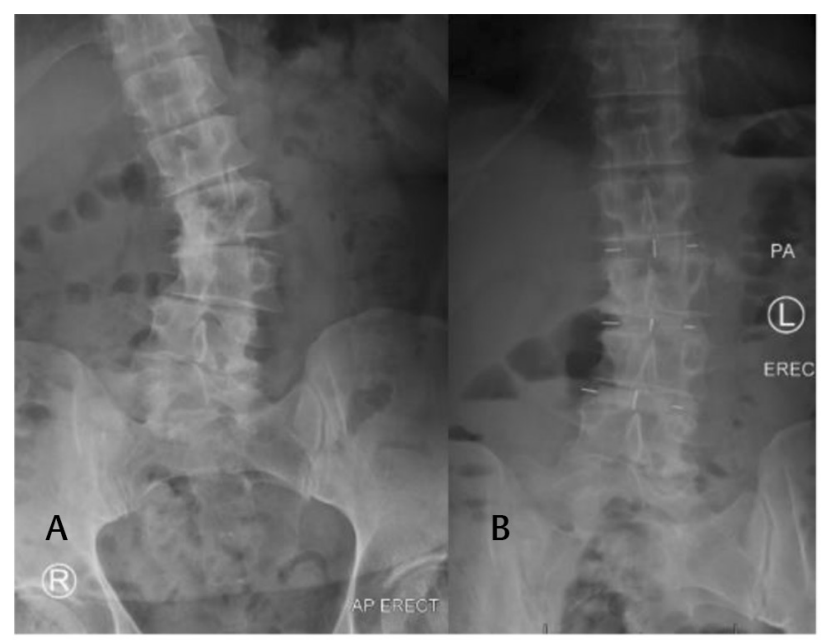

Fig. 1 Preoperative (A) and postoperative (B) anteroposterior radiographs of the lumbar spine in a patient treated with LLIF with significant coronal realignment. LLIF, lateral lumbar interbody fusion.

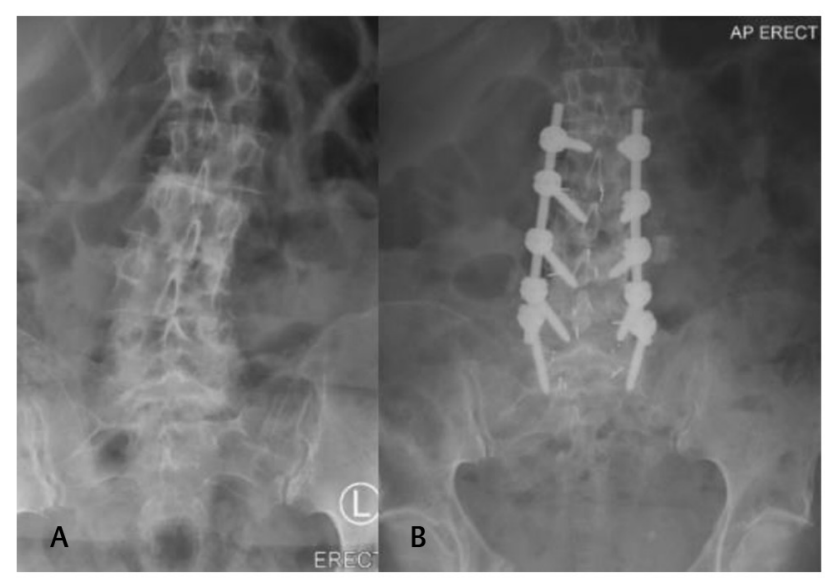

Fig. 2 Preoperative (A) and postoperative (B) anteroposterior radiographs of the lumbar spine in a patient treated with LLIF with pedicle screws and rods.

Table 1 Characteristics of patients treated with LLIF

\begin{tabular}{|l|l|}
\hline Age $(y)$ & average 61; range 42-80 \\
\hline Sex & 22 females; 10 males \\
\hline Smokers & 11 \\
\hline BMI & average 29.25; range 20-38.5 \\
\hline
\end{tabular}

Abbreviations: BMI, body mass index; LLIF, lateral lumbar interbody fusion.

\section{Clinical Outcome Scores}

Validated clinical outcome scores were obtained on all patients preoperatively and at most recent follow-up. Outcome scores included the ODI. Complications were recorded as per the Clavien-Dindo classification of surgical complications and graded as any deviation from a normal postoperative course (Grade I) to needing more surgery down the track (Grade III). ${ }^{7}$

\section{Statistical Analysis}

Single specimen $t$-test was used to calculate clinical outcome scores. Statistical significance was defined as $p<0.05$. 

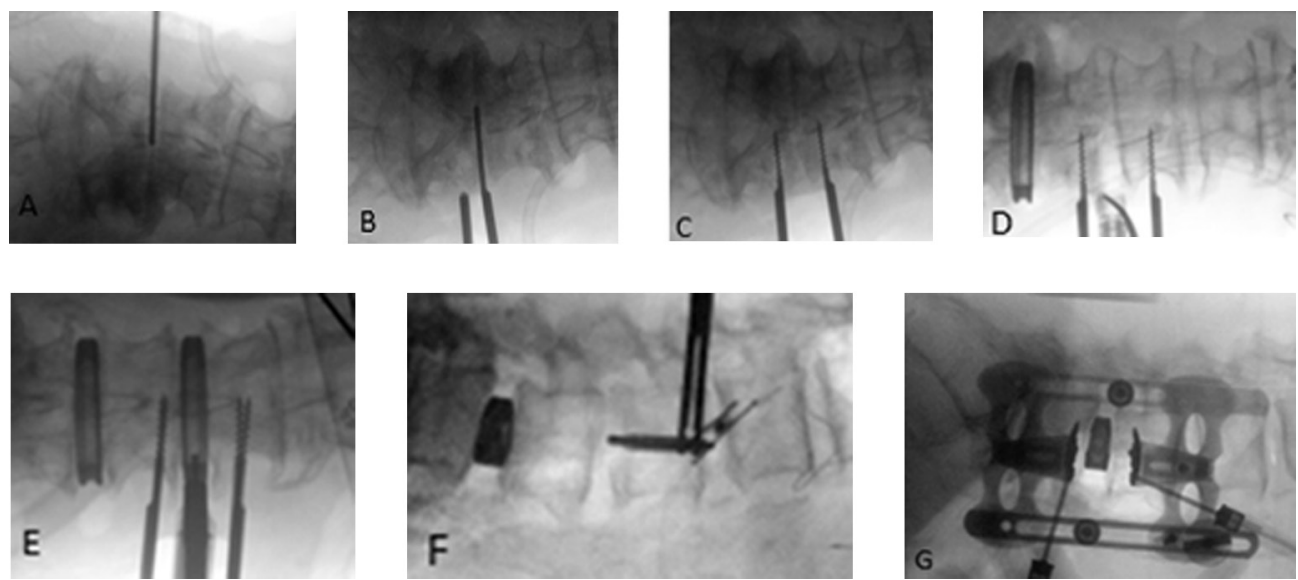

Fig. 3 Intraoperative images. Disc localization. (A) Distraction of disc space. (B), (C) Insertion of distractor pins. (D) Insertion of interbody cage. (E) Trans-psoas localization of the disc with neuromonitoring probe. (F) Ravine retractor system with distraction pins and interbody cage $(\mathbf{G})$.

\section{Results}

Our results showed that the ODI scores were lowered from a mean of 36.84 to $23.47 \%$ in patients undergoing LLIF. This was statistically significant result with $p$ value of $<0.05$. Our results also showed that the segmental coronal angle was corrected from 4.1 to 1.1 degrees (statistically significant with $p$ value of $<0.05$ ). Our short-term follow-up showed $100 \%$ fusion rate at all the levels amongst all the cases.

\section{Case Presentation}

A 66-year-old lady with history of chronic worsening lower back pain along with server right-sided sciatica was found to have advanced degenerative lumbar scoliosis. The ODI score was 41 preoperatively. Patient was neurologically not compromised and had all the preoperative workup including magnetic resonance imaging (MRI) lumbar spine, sagittal/coronal balance X-rays, bone mineral density check, and dynamic X-rays.

MRI scan showed multilevel disc degenerative disease and multilevel (L1-S1) foraminal narrowing and neural impingement, mostly on the right side. X-rays revealed significant coronal imbalance, with convexity to the left worse at the L2/ L3 level (-Fig. 1A).

The patient underwent elective LLIF from L1-L4 as stage 1 and subsequently underwent posterior fixation with pedicle screws from L1-S1, with insertion of L5/S1 interbody cage, as stage 2, one week down the track.

The patient recovered well with clinically and statistically significant improvement of her posture, balance, pain, and independent functionality (postoperative ODI: 8). Postoperative imaging demonstrated good correction of coronal imbalance and complete fusion all the levels on subsequent follow-up down the track ( - Figs. 1B, 4).

\section{Postoperative Complications}

Of the 32 patients who underwent surgery, four patients (12\%) were noted to experience complications. Two patients had cage expulsion (polyetheretherketone [PEEK] cage) and required additional surgery. One patient experienced

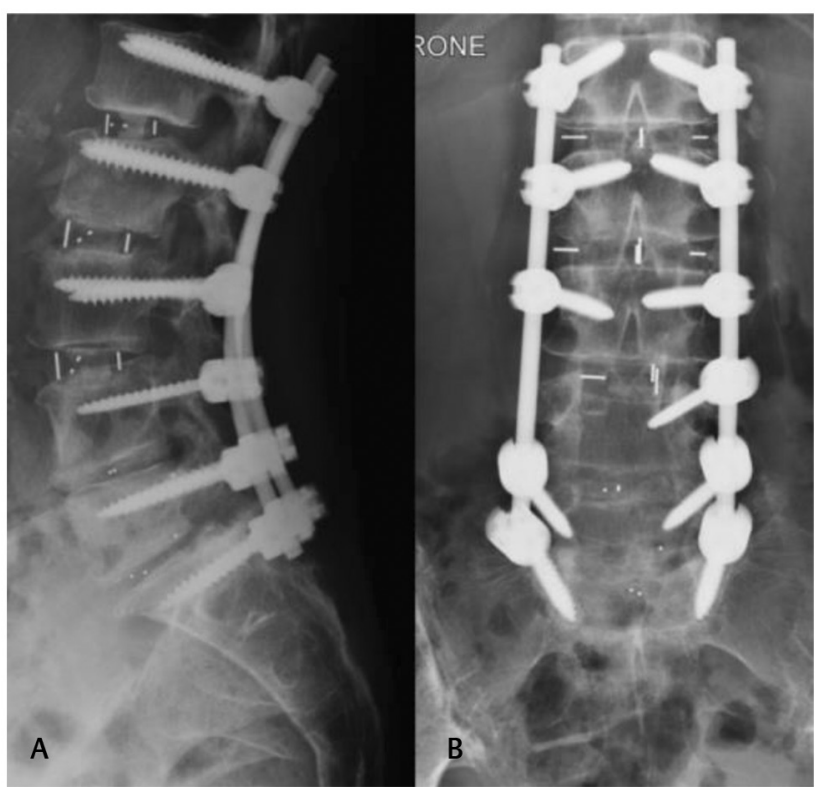

Fig. 4 Lateral (A) and anteroposterior (B) postoperative radiographs of the lumbar spine in a patient treated with LLIF with $360^{\circ}$ fusion and PLIF at bottom 2 levels. LLIF, lateral lumbar interbody fusion.

vertebral body fracture with loss of electromyography (EMG) signals and the procedure was abandoned; however, lateral fixation was performed without interbody fusion. This patient had postop foot weakness and required foot splint with some improvement of weakness on further follow-up. One patient had incisional lumbar hernia which is a very rare complication with this particular procedure and was managed conservatively.

\section{Discussion}

The LLIF is effective in preserving the anterior and posterior longitudinal ligaments and the facet joints. Therefore, LLIF procedure can maintain spinal alignment and stabilization due to ligamentotaxis. Moreover, the ability to insert a larger cage increases the size of the neural foramen. Thus, indirect 
foraminal decompression is possible. LLIF also has an excellent effect on the coronal and sagittal balance correction. ${ }^{8,9}$

Unlike other conventional posterior approaches, LLIF can provide strong mechanical stability by not only using a large interbody construct but also sparing ligamentous structures. It is also advantageous in avoiding dural tears, nerve root injury, facet joint damage, and paraspinal muscle injury. ${ }^{10}$ As compared with ALIF, LLIF is advantageous in that it can prevent the occurrence of injury to the abdominal internal organs as well as the peritoneal penetration, decrease risks of developing great vessel injury, and avoid an injury to the sympathetic chain. ${ }^{11}$

In the current study, we performed LLIF surgery at various levels, extending from L1 to L5. The LLIF approach can be made most easily at the L2-3-4 levels where such structures as the rib cage, the iliac crest, and lumbosacral plexus ${ }^{12}$ are less problematic. By contrast, it is difficult to approach the L4-L5 level because of such structures as the iliac crest and the lumbosacral plexus. The iliac crest can be avoided in patients with good position. In most of the patients where the iliac crest cannot be avoided, surgery can be successfully performed via a slightly oblique approach. ${ }^{12}$ There is a tendency for the lumbosacral plexus to pass the central region of the lateral aspect of the L4-L5 disc levels. ${ }^{13}$ Nerve damage can therefore be avoided through intraoperative neuromonitoring.

Acosta et $\mathrm{al}^{3}$ reported that the coronal segmental angle was corrected from 4.5 degree preoperatively to 1.5 degree postoperatively in patients undergoing LLIF. Consistent with previous reports, our results also showed that the segmental coronal angle was corrected from 4.1 to 1.1 degree. These findings have been consistently reported in studies about LLIF, ${ }^{3,14}$ thus indicating that LLIF is effective for the correction of coronal deformity.

LLIF is a minimally-invasive surgery, but it is disadvantageous in that autologous bone cannot be harvested. Therefore, bone prosthesis should be mainly used for LLIF. To overcome this disadvantage, we used i-Factor bone graft (an organic bone material) for all of our patients with good results.

The incidence of complications due to LLIF varies, ranging from 0.7 to $62.7 \% .{ }^{15-17}$ Such complications include psoas muscle injury and edema due to a retroperitoneal transpsoas approach, leading to hip flexor weakness, thigh/groin pain and numbness due to genitofemoral nerve injury, meralgia paresthetica due to lateral femoral cutaneous nerve injury, and numbness due to nerve root injury or lumbosacral plexus injury. ${ }^{18,19}$ Our results showed that the overall incidence of complications was $12 \%$ during a follow-up period of 1 year.

The limitations of the current study are that it enrolled a small number of patients with short term follow-up. Our results cannot therefore be generalized. Further large-scale, long-term follow-up studies are warranted to overcome the above limitations. According to one report concerning the size of neural foramen following LLIF, the foraminal area was increased by approximately $35 \% .{ }^{20}$ We did not include assessment of neural foramen in our study and consider it as another limitation of our study. It is recommended that a substantially long cage ${ }^{21}$ be used, so that it may be trapped in the apophysis ring during LLIF. This is because the apophysis is the most powerful structure in the vertebral body and it is useful to reduce subsidence and maintain disc height. ${ }^{22}$ We did not include these measurements in our study due to shorter follow-up. Johnson et al. ${ }^{14}$ reported that the segmental lumbar lordosis was significantly increased from 3.0 to 6.6 degrees following LLIF, but there was a nonstatistically significant change in the regional lumbar lordosis. Other studies have reported that LLIF was more effective in forming segmental lordosis when the cage was anteriorly inserted, thus indicating that the position of cage might affect the lumbar lordosis. ${ }^{23}$ We will include lordotic measurements in our second stage study.

\section{Conflict of Interest}

S. A., A. D., and A. K. D. declared no conflict of interests. C. S. has no conflict of interest related to K2M products or Cerapedics products used in this study; however, royalties were received from $\mathrm{K} 2 \mathrm{M}$ for an unrelated product.

\section{References}

1 McAfee PC, Regan JJ, Geis WP, Fedder IL. Minimally invasive anterior retroperitoneal approach to the lumbar spine. Emphasis on the lateral BAK. Spine 1998;23(13):1476-1484

2 Youssef JA, McAfee PC, Patty CA, et al. Minimally invasive surgery: lateral approach interbody fusion: results and review. Spine 2010;35(26, Suppl):S302-S311

3 Acosta FL, Liu J, Slimack N, Moller D, Fessler R, Koski T. Changes in coronal and sagittal plane alignment following minimally invasive direct lateral interbody fusion for the treatment of degenerative lumbar disease in adults: a radiographic study. J Neurosurg Spine 2011;15(1):92-96

4 Dakwar E, Cardona RF, Smith DA, Uribe JS. Early outcomes and safety of the minimally invasive, lateral retroperitoneal transpsoas approach for adult degenerative scoliosis. Neurosurg Focus 2010;28(3):E8

5 Sharma AK, Kepler CK, Girardi FP, Cammisa FP, Huang RC, Sama AA. Lateral lumbar interbody fusion: clinical and radiographic outcomes at 1 year: a preliminary report. J Spinal Disord Tech 2011;24(4):242-250

6 Vanderpool DW, James JI, Wynne-Davies R. Scoliosis in the elderly. J Bone Joint Surg Am 1969;51(3):446-455

7 Clavien PA, Barkun J, de Oliveira ML, et al. The Clavien-Dindo classification of surgical complications: five-year experience. Ann Surg 2009;250(2):187-196

8 Cappuccino A, Cornwall GB, Turner AW, et al. Biomechanical analysis and review of lateral lumbar fusion constructs. Spine 2010;35(26, Suppl):S361-S367

9 Dooris AP, Goel VK, Grosland NM, Gilbertson LG, Wilder DG. Load-sharing between anterior and posterior elements in a lumbar motion segment implanted with an artificial disc. Spine 2001;26(6):E122-E129

10 Oliveira L, Marchi L, Coutinho E, Pimenta L. A radiographic assessment of the ability of the extreme lateral interbody fusion procedure to indirectly decompress the neural elements. Spine 2010;35(26, Suppl):S331-S337

11 Laws CJ, Coughlin, DG, Lotx. . JC, Serhan, HA, Hu, SS. Spine 2012:37:819-825 
12 Ozgur BM, Aryan HE, Pimenta L, Taylor WR. Extreme lateral interbody fusion (XLIF): a novel surgical technique for anterior lumbar interbody fusion. Spine J 2006;6(4):435-443

13 Moro T, Kikuchi S, Konno S, Yaginuma H. An anatomic study of the lumbar plexus with respect to retroperitoneal endoscopic surgery. Spine 2003;28(5):423-428

14 Johnson RD, Valore A, Villaminar A, Comisso M, Balsano M. Pelvic parameters of sagittal balance in extreme lateral interbody fusion for degenerative lumbar disc disease. J Clin Neurosci 2013;20(4):576-581

15 Cummock MD, Vanni S, Levi AD, Yu Y, Wang MY. An analysis of postoperative thigh symptoms after minimally invasive transpsoas lumbar interbody fusion. J Neurosurg Spine 2011; 15(1):11-18

16 Knight RQ, Schwaegler P, Hanscom D, Roh J. Direct lateral lumbar interbody fusion for degenerative conditions: early complication profile. J Spinal Disord Tech 2009;22(1):34-37

17 Rodgers WB, Gerber EJ, Patterson J. Intraoperative and early postoperative complications in extreme lateral interbody fusion: an analysis of 600 cases. Spine 2011;36(1):26-32
18 Houten JK, Alexandre LC, Nasser R, Wollowick AL. Nerve injury during the transpsoas approach for lumbar fusion. J Neurosurg Spine 2011;15(3):280-284

19 Moller DJ, Slimack NP, Acosta FL Jr, Koski TR, Fessler RG, Liu JC. Minimally invasive lateral lumbar interbody fusion and transpsoas approach-related morbidity. Neurosurg Focus 2011;31(4):E4

20 Kepler CK, Sharma AK, Huang RC, et al. Indirect foraminal decompression after lateral transpsoas interbody fusion. J Neurosurg Spine 2012;16(4):329-333

21 Le TV, Baaj AA, Dakwar E, et al. Subsidence of polyetheretherketone intervertebral cages in minimally invasive lateral retroperitoneal transpsoas lumbar interbody fusion. Spine 2012;37(14): 1268-1273

22 Hollowell JP, Vollmer DG, Wilson CR, Pintar FA, Yoganandan N. Biomechanical analysis of thoracolumbar interbody constructs. How important is the endplate? Spine 1996;21(9):1032-1036

23 Kepler CK, Huang RC, Sharma AK, et al. Factors influencing segmental lumbar lordosis after lateral transpsoas interbody fusion. Orthop Surg 2012;4(2):71-75 